\title{
High strain-rate compressive behavior and constitutive modeling of selected polymers
}

\author{
K. Nakai and T. Yokoyama \\ Department of Mechanical Engineering, Okayama University of Science, Okayama 700-0005, Japan
}

\begin{abstract}
The present paper deals with constitutive modeling of the compressive stress-strain behavior of selected polymers at strain rates from $10^{-3}$ to $10^{3} / \mathrm{s}$ using a modified Ramberg-Osgood equation. High strain-rate compressive stress-strain curves for four different commercially available extruded polymers are determined on the standard split Hopkinson pressure bar. The low and intermediate strain-rates compressive stress-strain relations are measured in an Instron testing machine. The five parameters for the modified Ramberg-Osgood equation are determined by fitting to the experimental compressive stress-strain data using a least-squares fit. The compressive stress-strain curves at three different strain rates derived from the modified Ramberg-Osgood models are compared with the experimental results. It is shown that the compressive stress-strain behavior during loading process can be successfully predicted by the modified Ramberg-Osgood equation.
\end{abstract}

\section{Introduction}

Polymeric materials with low mechanical impedance have been extensively used as one of structural materials for aircraft, automotive and electronic components. These components are often subjected to impact loading in service environments. Therefore, it is needed to characterize the impact mechanical behavior of polymeric materials. To date, the impact compressive [1-4], tensile [5-8] and torsional $[7,9]$ stress-strain properties of several polymers have been determined with the conventional [10] or modified split Hopkinson pressure bar (SHPB). The effects of strain rate and temperature on the compressive characteristics of several polymers were examined using a drop-weight apparatus [11-13]. In order to accurately describe the high strain-rate stress-strain behavior of the polymers, it is required to develop their strain-rate dependent constitutive equations. Many complicated constitutive models such as nonlinear viscoelastic-plastic models [14-16], modified Johnson-Cook model [17], modified Zerilli-Armstrong model [18] and nonlinear power law model [19] have been proposed to predict the stress-strain behavior of the polymers over a wide range of strain rates and temperatures. However, it is very difficult to perform simulations of the response of polymeric structures to dynamic loading using these models with a large number of parameters.

The purpose of the present paper is to model the compressive stress-strain behavior of the selected polymers at strain rates from $10^{-3}$ to $10^{3} / \mathrm{s}$ using a simple strain-rate dependent constitutive equation. Four different commercially available extruded polymers or PA-6 (Polyamide-6), PA-66 (Polyamide-66), PC (Polycarbonate) and POM (Polyoxymethylene) were tested at room temperature. Cylindrical specimens with a slenderness ratio $l / d$ (= length/diameter) of 0.5 were used in the SHPB tests, and those with $l / d=1.5$ were used in the low and intermediate strain-rate tests. The compressive stress-strain loops at strain rates up to about $10^{3} / \mathrm{s}$ were determined in the conventional SHPB. The compressive stress-strain loops at low and intermediate strain rates were measured with an Instron 5500R testing machine. The strain-rate dependent Ramberg-Osgood equation was applied to model the experimental compressive stress-strain loops over a wide range of strain rates.

\section{Experimental procedure}

\subsection{Test polymers and specimen preparation}

Four different common polymers, i.e., three semicrystalline polymers: PA-6, PA-66 (Toyo Plastic Seiko Co., Ltd., Tokyo, Japan), POM (Polyplastics Co., Ltd., Tokyo, Japan) and an amorphous polymer: PC (Toyo Plastic Seiko Co., Ltd., Tokyo, Japan) were chosen. Cylindrical specimens were machined out of commercial extruded rods with a diameter of nearly $10 \mathrm{~mm}$ into short cylinders with a diameter of $9 \mathrm{~mm}$. The specimen end surfaces were carefully polished with waterproof abrasive paper (\#1500). The static specimen's length was determined to be $l / d=1.5$ $(l \fallingdotseq 13.5 \mathrm{~mm}, d=9 \mathrm{~mm})$ in accordance with the ASTM Designation E9-89a [20] (see, Table 1). The slenderness ratio $l / d$ of the impact specimen (see, Table 2) was taken as $0.5(l \fallingdotseq 4.5 \mathrm{~mm}, d=9 \mathrm{~mm})$, falling in an appropriate slenderness ratio range between 0.5 and 1.0 suggested by Gray [21] in the conventional SHPB tests. All specimens of the different four polymers were tested in the as-received state.

\subsection{Low and intermediate strain-rate compression testing}

The low and intermediate strain rate compression tests were conducted on the cylindrical specimens with $l / d=$ 1.5 using the Instron 5500R testing machine at crosshead speeds of 1 and $100 \mathrm{~mm} / \mathrm{min}$, respectively. The specimens were loaded up to a given strain and unloaded at the same crosshead speed. Both ends of the specimen were lubricated with petroleum jelly (white Vaseline) to minimize frictional restraints between the specimen and the loading anvils. 
Table 1. Geometry of static compression specimen.

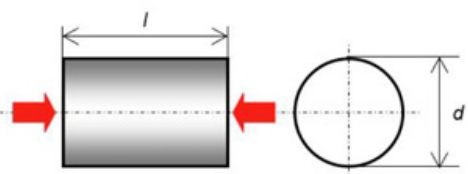

\begin{tabular}{c|ccc}
\hline Polymer & $\begin{array}{c}\text { Length } \\
I(\mathrm{~mm})\end{array}$ & $\begin{array}{c}\text { Diameter } \\
d(\mathrm{~mm})\end{array}$ & $\begin{array}{c}\text { Slenderness ratio } \\
/ / d\end{array}$ \\
\hline PA-6 & $13.53 \pm 0.02$ & 9.05 & 1.5 \\
\hline PA-66 & $13.51 \pm 0.02$ & 9.01 & 1.5 \\
\hline PC & $13.45 \pm 0.02$ & 8.97 & 1.5 \\
\hline POM & $13.58 \pm 0.02$ & 9.05 & 1.5 \\
\hline
\end{tabular}

Table 2. Geometry of impact compression specimen.

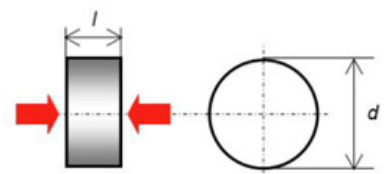

\begin{tabular}{c|ccc}
\hline Polymer & $\begin{array}{c}\text { Length } \\
/(\mathrm{mm})\end{array}$ & $\begin{array}{c}\text { Diameter } \\
d(\mathrm{~mm})\end{array}$ & $\begin{array}{c}\text { Slenderness ratio } \\
/ / d\end{array}$ \\
\hline PA-6 & $4.46 \pm 0.03$ & 9.05 & 0.5 \\
\hline PA-66 & $4.51 \pm 0.02$ & 9.01 & 0.5 \\
\hline PC & $4.50 \pm 0.02$ & 9.00 & 0.5 \\
\hline POM & $4.52 \pm 0.02$ & 9.04 & 0.5 \\
\hline
\end{tabular}

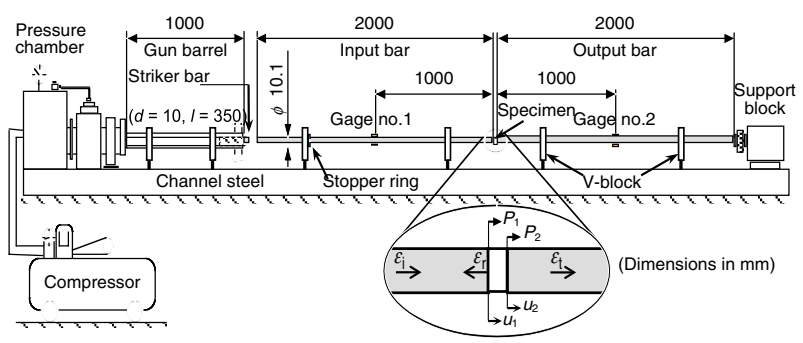

Fig. 1. Schematic diagram of conventional split Hopkinson pressure bar set-up (associated recording system not shown).

\subsection{Split Hopkinson bar testing}

The general arrangement of the SHPB set-up is given in Fig. 1. The SHPB set-up consists of two 2024-T4 Al alloy bars of $2000 \mathrm{~mm}$ in length and $10.1 \mathrm{~mm}$ in diameter, which remain elastic during the tests. A striker bar made of the same material is $350 \mathrm{~mm}$ in length and $10.1 \mathrm{~mm}$ in diameter. The mechanical properties of the 2024-T4 Al alloy are as follows: Young's modulus $E=73 \mathrm{GPa}$; longitudinal elastic wave velocity $c_{\mathrm{o}}=5130 \mathrm{~m} / \mathrm{s}$; mechanical impedance $Z=\rho c_{\mathrm{o}}=14.2 \times 10^{6} \mathrm{~kg} / \mathrm{m}^{2} \mathrm{~s}$; yield strength $\sigma_{\mathrm{Y}}=450 \mathrm{MPa}$. The $\mathrm{Al}$ alloy bars with low mechanical impedance are used to reduce a drastic impedance mismatch between the polymer specimen $\left(Z \fallingdotseq 1 \sim 2 \times 10^{6} \mathrm{~kg} / \mathrm{m}^{2} \mathrm{~s}\right)$ and the conventional steel bars $\left(Z \fallingdotseq 40 \times 10^{6} \mathrm{~kg} / \mathrm{m}^{2} \mathrm{~s}\right)$, which results in a transmitted strain signal with a very low signal-to-noise ratio. The specimen is held in place between the input and output bars by applying a very small pre-compression load with turning of the head of a support block. As in the static tests, lubricant (or petroleum jelly) is applied to the bar/specimen interfaces to reduce the

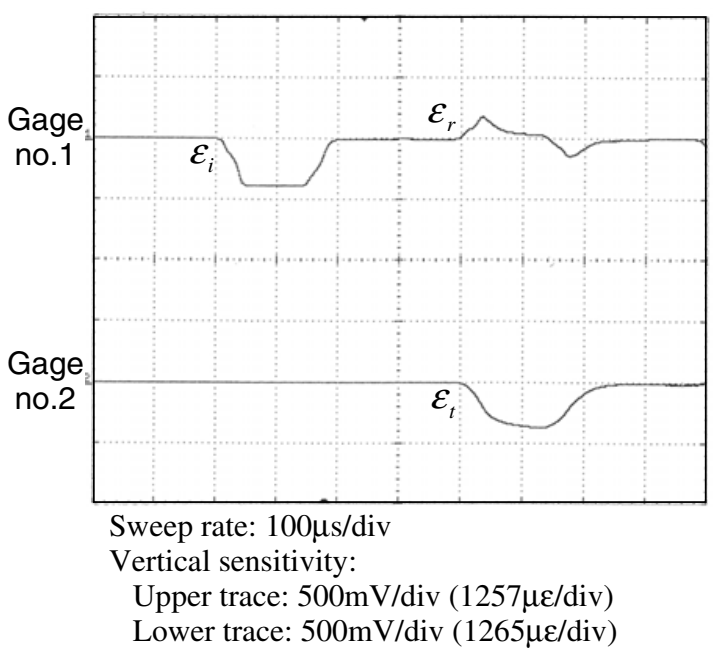

Fig. 2. Oscilloscope traces from SHPB test on $\mathrm{PC}\left(V_{\mathrm{S}}=\right.$ $9.3 \mathrm{~m} / \mathrm{s})$.

frictional effects. A pulse shaping technique [22] is used to generate well-defined compressive strain pulses without higher frequency components in the input bar. Namely, a $0.2 \mathrm{~mm}$ thick $1050 \mathrm{Al}$ disk of nearly $10 \mathrm{~mm}$ in diameter is attached onto the impact (left) end of the input bar using a thin layer of petroleum jelly. Details of the test procedure can be found elsewhere [23].

From the elementary one-dimensional theory of elastic wave propagation, we can determine the nominal strain $\varepsilon(t)$, strain rate $\dot{\varepsilon}(t)$ and stress $\sigma(t)$ in the specimen from the SHPB test records as [24]

$$
\begin{gathered}
\varepsilon(t)=\frac{u_{1}(t)-u_{2}(t)}{l}=\frac{2 c_{\mathrm{o}}}{l} \int_{0}^{t}\left\{\varepsilon_{i}\left(t^{\prime}\right)-\varepsilon_{t}\left(t^{\prime}\right)\right\} \mathrm{d} t^{\prime} \\
\dot{\varepsilon}(t)=\frac{\dot{u}_{1}(t)-\dot{u}_{2}(t)}{l}=\frac{2 c_{\mathrm{o}}}{l}\left\{\varepsilon_{i}(t)-\varepsilon_{t}(t)\right\} \\
\sigma(t)=\frac{P_{2}(t)}{A_{\mathrm{S}}}=\frac{A E}{A_{\mathrm{S}}} \varepsilon_{t}(t)
\end{gathered}
$$

Here $u$ and $P$ are the displacement and the axial force on both ends of the specimen, respectively, (where subscripts 1 and 2 denote the left and right interfaces, respectively; see the inset in Fig. 1); $A, E$ and $c_{\mathrm{o}}$ are the crosssectional area, Young's modulus and the longitudinal elastic wave velocity of the Hopkinson (2024-T4 Al alloy) bars; $A_{\mathrm{S}}$ is the cross-sectional area of the specimen. Equations (1) to (3) are derived under the assumption of dynamic force equilibrium across the specimen, which can be expressed as

$$
P_{1}(t)=P_{2}(t) \quad \text { or } \quad \varepsilon_{i}(t)+\varepsilon_{r}(t)=\varepsilon_{t}(t)
$$

where

$$
P_{1}(t)=A E\left[\varepsilon_{i}(t)+\varepsilon_{r}(t)\right], \quad P_{2}(t)=A E \varepsilon_{t}(t)
$$

In the above derivations, the incident and reflected strain pulses are time-shifted to the specimen-input bar interface, and the transmitted strain pulse is time-shifted to the specimen-output bar interface. Eliminating time $t$ 


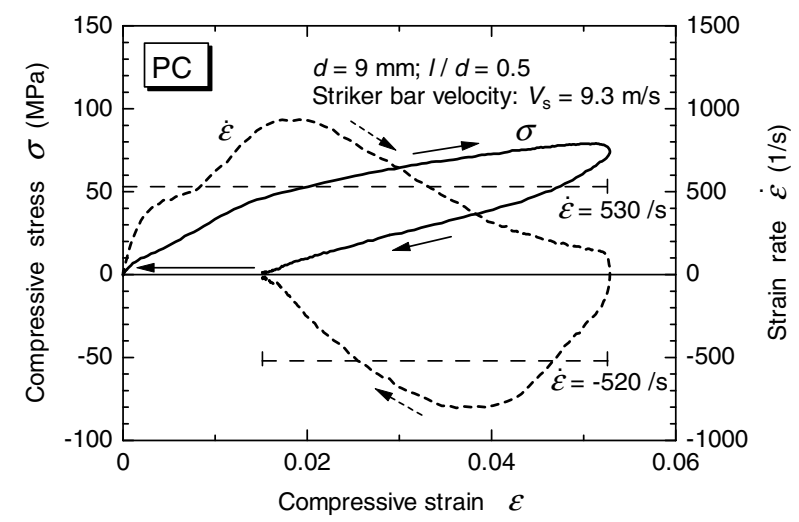

Fig. 3. Dynamic stress-strain loop and strain rate-strain relation in compression for PC.

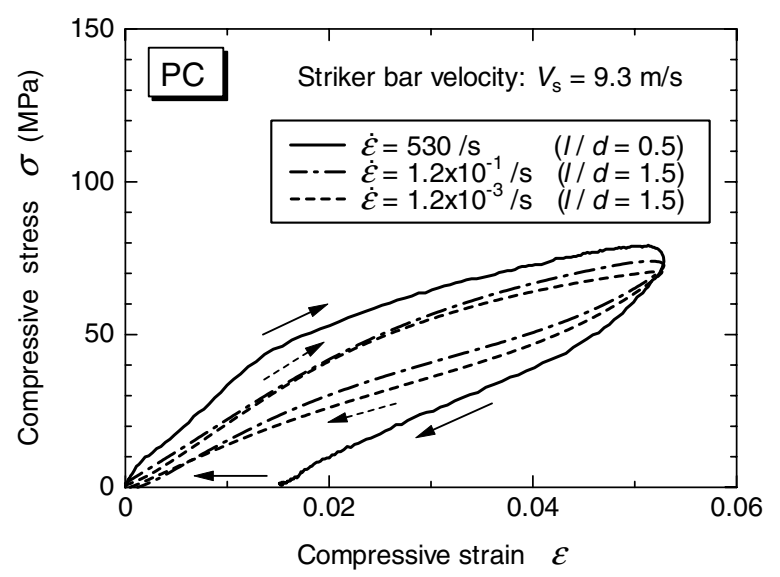

Fig. 4. Compressive stress-strain loops for PC at three different strain rates.

through Eqs. (1) to (3) yields the nominal (or engineering) compressive stress-strain and strain rate-strain relations. In this work, the compressive stress and strain are taken as positive.

\section{Results and discussion}

A number of the SHPB tests were conducted on the four different polymers at room temperature. Figure 2 indicates typical oscilloscope records from the SHPB test on PC. The top trace gives the incident and reflected strain pulses ( $\varepsilon_{i}$ and $\varepsilon_{r}$ ), and the bottom trace gives the strain pulse $\left(\varepsilon_{t}\right)$ transmitted through the specimen. The recorded signal data are neither smoothed nor averaged electronically. It is very important to notice that the duration $(\fallingdotseq 260 \mu \mathrm{s})$ of the reflected and transmitted strain pulses is longer than that $(\fallingdotseq 200 \mu$ s) of the incident strain pulse. This is due to a very long retardation time [25] of the polymers. Figure 3 presents the resulting dynamic stress-strain loop and strain rate-strain relation in compression. The strain rate does not remain constant during loading as well as unloading, and hence the strain rate $\dot{\varepsilon}=530 /$ s given denotes the average one during loading process, which is calculated by dividing the area under the strain ratestrain curve up to the maximum strain $(\fallingdotseq 0.053)$ by the value of its strain. As in the low and intermediate strain

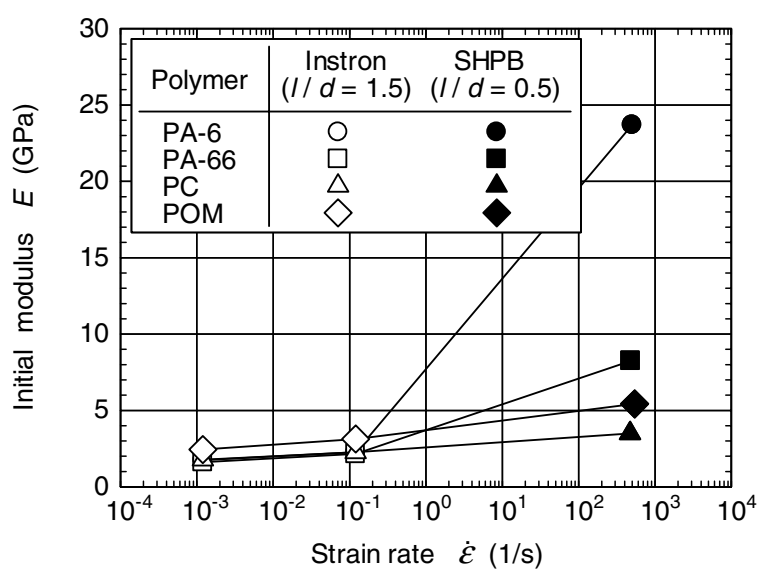

Fig. 5. Effect of strain rate on initial modulus for four different polymers.

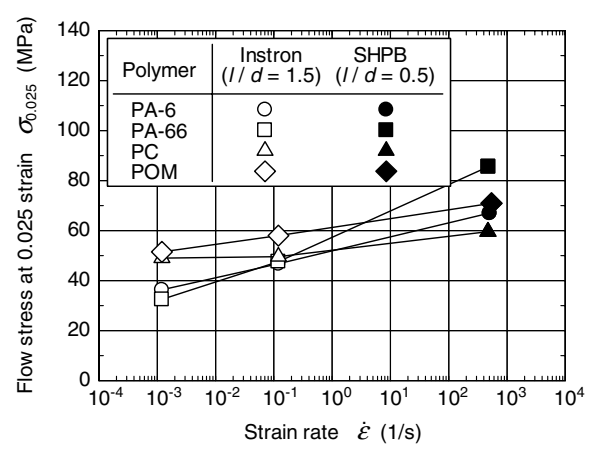

Fig. 6. Effect of strain rate on flow stress at 0.025 strain for four different polymers.

rate tests, the dynamic stress-strain loop is not closed, and, consequently, a residual strain of about 0.015 is gradually recovered to zero in time. Figure 4 shows the compressive stress-strain loops of PC at three different strain rates. The initial slope (or initial modulus $E$ ), the flow stress and the area within the loop increase greatly with increasing strain rate. In order to evaluate the effect of strain rate on the compressive properties of the four different polymers, the measured values for the initial modulus (defined as the secant modulus at 0.002 strain) and flow stress at a given strain of 0.025 are plotted in Figures 5 and 6, respectively, as functions of the average strain rate $\dot{\varepsilon}$ during loading process. It can be seen that the initial modulus and flow stress increase significantly with increasing strain rate for all polymers. All polymers exhibit inherent dynamic viscoelastic characteristics.

In an attempt to quantitatively evaluate the rate dependence of the flow stress at 0.025 strain, two different strainrate sensitivity parameters $\beta$ and $m$ [26] are introduced. The two parameters estimated for all four polymers are summarized in Table 3, where $\sigma_{1}$ and $\sigma_{2}$ are stresses at the average strain rates $\dot{\varepsilon}_{1}$ and $\dot{\varepsilon}_{2}$, respectively, for a fixed strain of 0.025 . The choice of 0.025 strain is made between the common strains at strain rates corresponding approximately to the respective average strain rates during loading. It is seen that the flow stress of PA-66 displays the highest strain-rate sensitivity, whereas that of PC exhibits the lowest strain-rate dependence. A similar trend 
Table 3. Strain-rate sensitivity parameters for four different polymers within a range of strain rates from $\dot{\varepsilon}_{1}=1.2 \times 10^{-3} / \mathrm{s}$ to $\dot{\varepsilon}_{2} \fallingdotseq 500 / \mathrm{s}$.

\begin{tabular}{c|cc}
\hline Polymer & $\beta=\left.\frac{\sigma_{2}-\sigma_{1}}{\log \left(\dot{\varepsilon}_{2} / \dot{\varepsilon}_{1}\right)}\right|_{\begin{array}{c}(\mathrm{MPa}) \\
\varepsilon=0.025\end{array}}$ & $m=\left.\frac{\log \left(\sigma_{2} / \sigma_{1}\right)}{\log \left(\dot{\varepsilon}_{2} / \dot{\varepsilon}_{1}\right)}\right|_{(1 / 1)}$ \\
& $\dot{\varepsilon}_{2}>\dot{\varepsilon}_{1}$ & $\dot{\varepsilon}_{2}>\dot{\varepsilon}_{1}$ \\
\hline PA-6 & $5.5(5.6)^{*}$ & 0.047 \\
\hline PA-66 & $10(5.9)$ & 0.079 \\
\hline PC & $1.8(3.48)$ & 0.014 \\
\hline POM & $3.5(\mathrm{NA})$ & 0.025 \\
\hline * Note: values in parentheses are taken from Ref [4] $(\varepsilon=0.10)$
\end{tabular}

${ }^{*}$ Note: values in parentheses are taken from Ref.[4] $(\varepsilon=0.10)$

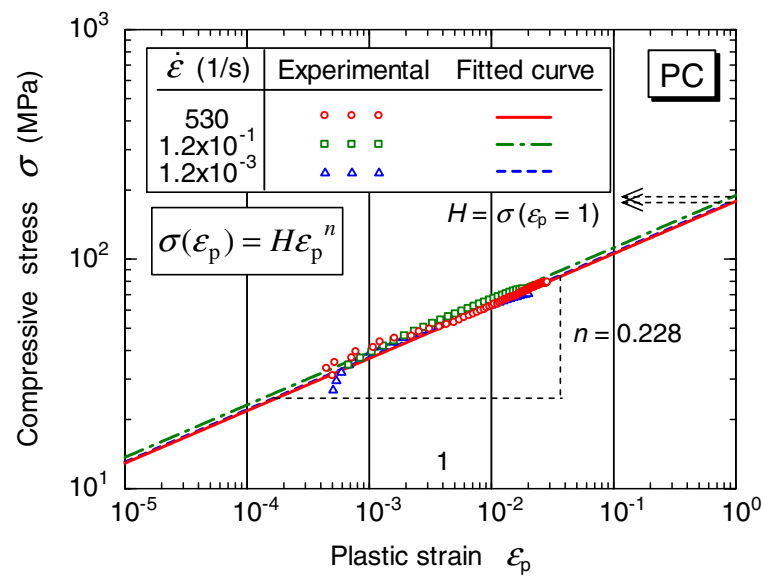

Fig. 7. Determination of constants $H$ and $n$ in Eq. (6).

in the values for the strain-rate sensitivity parameter $\beta$ is observed between the present work and Ref. [4].

\section{Constitutive equations}

In an effort to model the compressive stress-strain behavior of the four different polymers, the Ramberg-Osgood equation [27] is used, i.e.,

$$
\varepsilon(\sigma)=\frac{\sigma}{E}+\left(\frac{\sigma}{H}\right)^{\frac{1}{n}}
$$

where $E$ is the initial (or Young's) modulus, $H$ the strength coefficient and $n$ the strain hardening exponent. The first and second terms on the right hand side of Eq. (5) correspond to the elastic strain $\varepsilon_{e}$ and the plastic strain $\varepsilon_{p}$, respectively. We can obtain the following relation from Eq. (5):

$$
\sigma\left(\varepsilon_{\mathrm{p}}\right)=H \varepsilon_{\mathrm{p}}^{n}=H\left(\varepsilon-\frac{\sigma}{E}\right)^{n}
$$

Taking logarithms of both sides of Eq. (6) gives

$$
\log \sigma=\log H+n \log \varepsilon_{\mathrm{p}}
$$

Figure 7 shows typical $\log$-log plots of the stress $\sigma$-plastic strain $\varepsilon_{\mathrm{p}}$ data for $\mathrm{PC}$ at three different strain rates obtained from Fig. 4. The constants $H$ and $n$ are determined by fitting Eq. (7) to the data points on the $\sigma-\varepsilon_{\mathrm{p}}$ curves using a least-squares fit. The constant $H$ corresponds to the value of $\sigma$ at $\varepsilon_{\mathrm{p}}=1$. The constant $n$

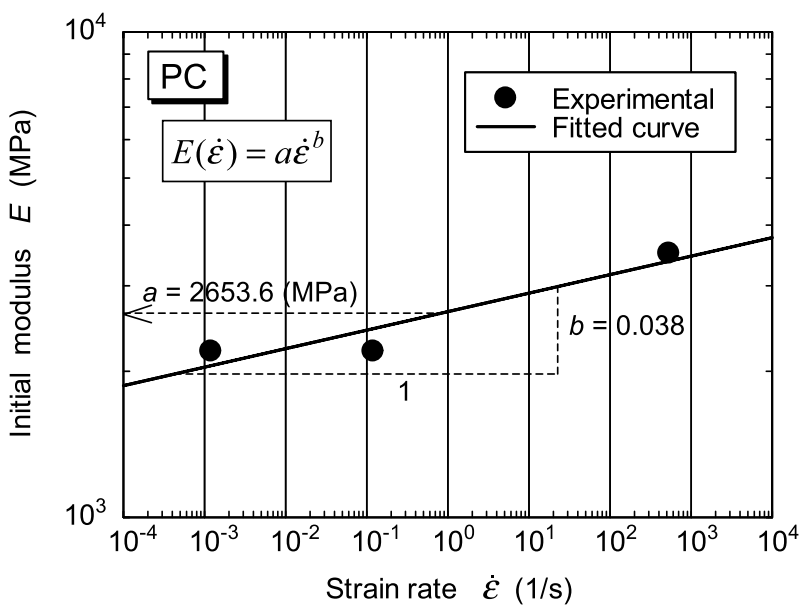

Fig. 8. Initial modulus $E$ as function of strain rate $\dot{\varepsilon}$ for PC. Determination of constants $a$ and $b$ in modified Ramberg-Osgood equation.

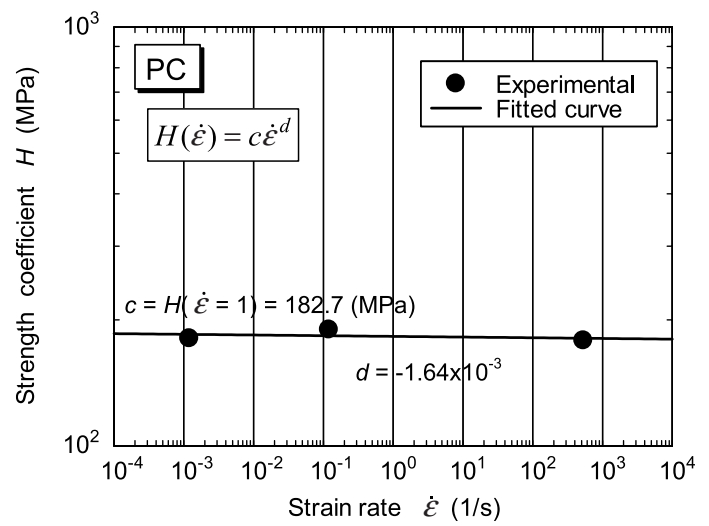

Fig. 9. Strength coefficient $H$ as function of strain rate $\dot{\varepsilon}$ for PC. Determination of constants $c$ and $d$ in modified Ramberg-Osgood equation.

is given as the slope of the linear fit line. As can be seen from Fig. 7, $n$ is independent of strain rate up to 530/s, whereas $H$ depends on the strain rate. Both $E$ and $H$ are given as simple functions of strain rate as

$$
E(\dot{\varepsilon})=a \dot{\varepsilon}^{b} ; \quad H(\dot{\varepsilon})=c \dot{\varepsilon}^{d}
$$

Taking logarithms of both sides of Eq. (8) leads to

$$
\log E=\log a+b \log \dot{\varepsilon} ; \quad \log H=\log c+d \log \dot{\varepsilon}
$$

Substituting Eq. (8) into Eq. (5), we have the strainrate dependent Ramberg-Osgood equation form (modified Ramberg-Osgood equation) [28] as

$$
\varepsilon(\sigma, \dot{\varepsilon})=\frac{\sigma}{a \dot{\varepsilon}^{b}}+\left(\frac{\sigma}{c \dot{\varepsilon}^{d}}\right)^{\frac{1}{n}}
$$

The measured values for the initial modulus $E$ and the strength coefficient $H$ for PC are plotted in Figs. 8 and 9 , respectively, as functions of the average strain rate $\dot{\varepsilon}$ during loading process. The material constants $a, b, c$ and $d$ are obtained by performing the least-squares fit to the 

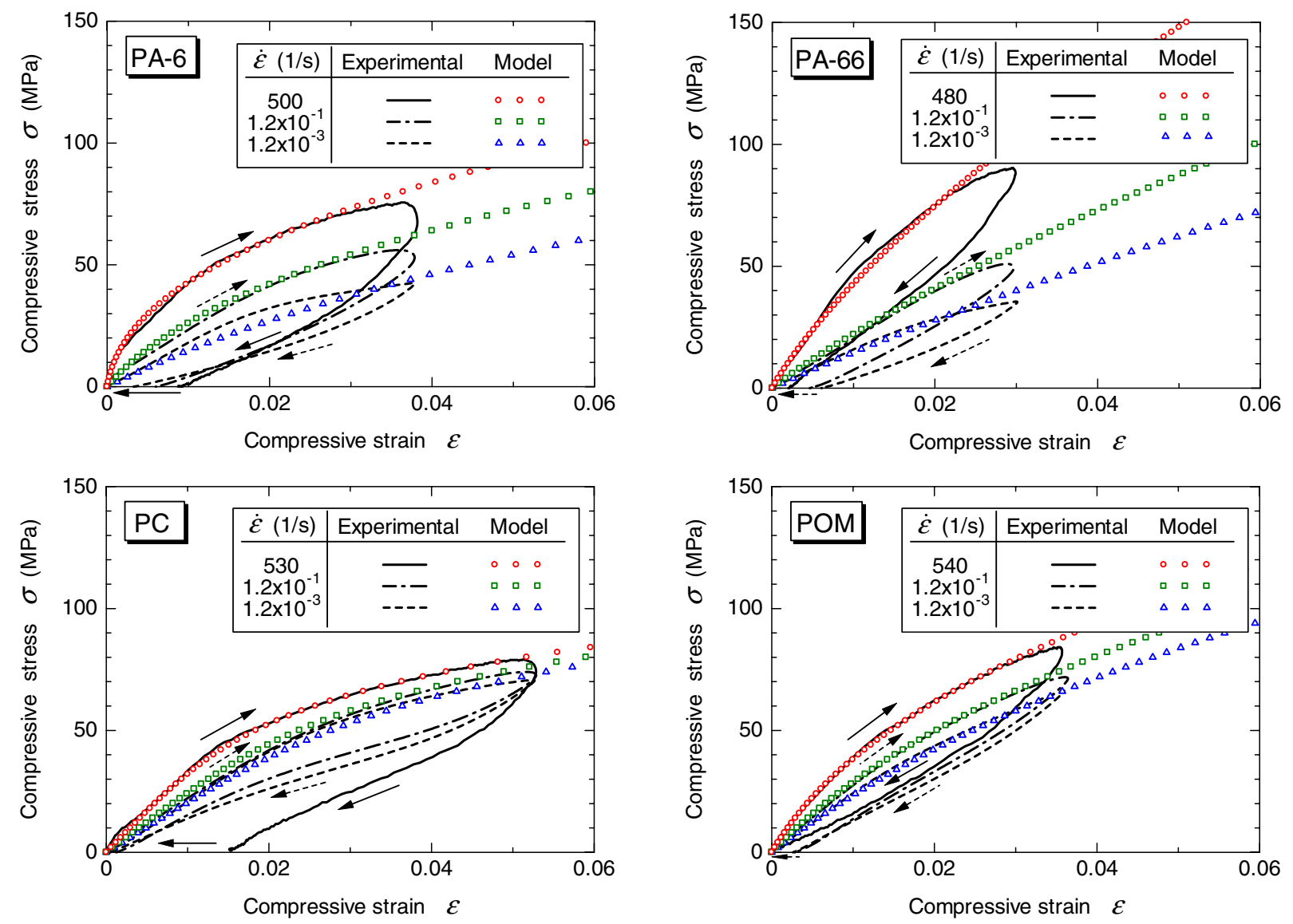

Fig. 10. Comparisons between measured compressive stress-strain curves and modified Ramberg-Osgood relations for four different polymers at three different strain rates.

Table 4. Parameter values for modified Ramberg-Osgood equation fitted to compressive stress-strain data for four different polymers.

\begin{tabular}{cccccc}
\hline Polymer & $a(\mathrm{MPa})$ & $b(-)$ & $c(\mathrm{MPa})$ & $d(-)$ & $n(-)$ \\
\hline PA-6 & 5152.2 & 0.172 & 334.8 & $9.19 \times 10^{-3}$ & 0.429 \\
\hline PA-66 & 3118.7 & 0.099 & 950.5 & $33.6 \times 10^{-3}$ & 0.556 \\
\hline PC & 2653.6 & 0.038 & 182.7 & $-1.64 \times 10^{-3}$ & 0.228 \\
\hline POM & 3754.6 & 0.055 & 486.7 & $1.06 \times 10^{-3}$ & 0.435 \\
\hline
\end{tabular}

measured data points (solid circles) using Eq. (9). The parameter values for Eq. (10) determined for the four different polymers are summarized in Table 4 . The fitted curves are shown as the solid lines in Figs. 8 and 9. The constants $b$ and $d$ are the strain-rate sensitivity exponents of the initial modulus and flow stress, respectively. A reasonable correlation can be found between the values for $d$ and the values for the two different strain-rate sensitivity parameters given in Table 3. Figure 10 depicts comparisons between the experimental compressive stress-strain curves and the modified Ramberg-Osgood relations for the four different polymers at three different strain rates. The compressive stress-strain behavior during loading process is accurately predicted by the modified Ramberg-Osgood equations for all four polymers.

\section{Conclusions}

The strain-rate dependence of the uniaxial compressive stress-strain loops for the four commercial polymers has been investigated with both the standard SHPB and the Instron 5500R testing machine. The constitutive modeling was performed using the modified Ramberg-Osgood equation. From the present study, we can draw the following conclusions:

(1) All four polymers exhibit intrinsic strain-rate dependent viscoelastic behavior and a high elastic aftereffect following complete unloading.

(2) The initial modulus and flow stress for all four polymers increase greatly with increasing strain rate.

(3) Of the four polymers, PA-6 shows the highest strainrate sensitivity of the initial modulus.

(4) Of the four polymers, PA-66 displays the highest strain-rate sensitivity of the flow stress.

(5) For all four polymers, the compressive stress-strain curves during loading process at strain rates from $10^{-3}$ to $10^{3} / \mathrm{s}$ can be successfully predicted by the modified Ramberg-Osgood equations.

(6) The modified Ramberg-Osgood equation cannot be applied to model the unloading stress-strain behavior at low or high strain rates. 


\section{References}

1. E.D.H. Davies and S.C. Hunter, Journal of the Mechanics and Physics of Solids, 11, 155 (1963).

2. S.S. Chiu and V.H. Neubert, Journal of the Mechanics and Physics of Solids, 15, 177 (1967).

3. E.W. Billington and C. Brissenden, International Journal of Mechanical Sciences, 13, 531 (1971).

4. S.M. Walley and J.E. Field, DYMAT Journal, 1, 211 (1994).

5. V.P.W. Shim, J. Yuan and S.-H. Lee, Experimental Mechanics, 41, 122 (2001).

6. W. Chen, F. Lu and M. Cheng, Polymer Testing, 21, 113 (2002).

7. A. Gilat, R.K. Goldberg and G.D. Roberts, Journal of Aerospace Engineering, 20, 75 (2007).

8. S. Fu, Y. Wang and Y. Wang, Polymer Testing, 28, 724 (2009)

9. N.A. Fleck, W.J. Stronge and J.H. Liu, Proceedings of the Royal Society of London, A429, 459 (1990).

10. H. Kolsky, Proceedings of the Physical Society, B62, 676 (1949).

11. H.P. Tardif and H. Marquis, Canadian Aeronautics and Space Journal, 9, 205 (1963).

12. B.J. Briscoe and I.M. Hutchings, Polymer, 17, 1099 (1976).

13. S. Hamdan and G.M. Swallowe, Journal of Materials Science, 31, 1415 (1996).

14. K.W. Chase and W. Goldsmith, Experimental Mechanics, 14, 10 (1974).

15. M.H. Sadd and D.H. Morris, Journal of Applied Polymer Science, 20, 421 (1976).
16. A.D. Mulliken and M.C. Boyce, International Journal of Solids and Structures, 43, 1331 (2006).

17. W. Chen and B. Zhou, Mechanics of Time-Dependent Materials, 2, 103 (1998).

18. J.L. Jordan, C.R. Siviour, J.R. Foley and E.N. Brown, Polymer, 48, 4184 (2007).

19. N.K. Naik, P.J. Shankar, V.R. Kavala, G. Ravikumar, J.R. Pothnis and H. Arya, Materials Science and Engineering A, 528, 846 (2011).

20. ASTM E9-89a, Annual Book of ASTM Standards (American Society for Testing and Materials, Philadelphia, 1995).

21. G.T. Gray III, ASM Handbook, Vol.8, Mechanical Testing and Evaluation (ASM International, Materials Park, OH, 2000).

22. B. Song and W. Chen, Experimental Mechanics, 44, 622 (2004).

23. T. Yokoyama and K. Nakai, Journal of the Japanese Society for Experimental Mechanics, Special Issue, 8, 98 (2008).

24. U.S. Lindholm, Journal of the Mechanics and Physics of Solids, 12, 317 (1964).

25. I.M. Ward and J. Sweeney, An Introduction to the Mechanical Properties of Solid Polymers, 2nd Edition (John Wiley \& Sons, Ltd, Chichester, 2004).

26. M. Malatyñski and J. Klepaczko, International Journal of Mechanical Sciences, 22, 173 (1980).

27. W. Ramberg and W.R. Osgood, Technical Note, No.902, National Advisory Committee for Aeronautics (Washington DC, 1943).

28. D.L. McLellan, AIAA Journal, 5, 446 (1967) 